\title{
ON THE TANGENTIAL PROPERTIES OF SURFACES
}

BY E. R. REIFENBERG

Communicated by E. J. McShane, January 9, 1962

It is the task of this paper to generalise certain results of [1] concerning the structure of surfaces. The motivation is in part to extend the solution [2] of the Plateau problem from the case of Hausdorff spherical measure to other forms of "area," particularly Hausdorff convex measure.

We consider the class of surfaces defined in [2]. $\mathrm{L} \subset \mathrm{H}_{m-1}(A)$ and $S$ is a surface with boundary $\supset L$. We write $\mu(L)$ for the minimum area $\Lambda^{m}(S-A)$ of surfaces with boundary $\supset L$. We also write $\mu(A)$ for $\mu\left(H_{m-1}(A)\right)$.

Let $S(P, r)$ denote the solid sphere of centre $P$ and radius $r$, and denote its surface by $s(P, r)$. Let $T$ be the set of points of $S^{0}=S-A$ where $S$ is $\left(\Lambda^{m}, m\right)$-restricted [3] and

$$
\frac{\mu(s(P, r) S)}{r^{m}} \rightarrow 0 .
$$

At these points we say there is an approximate tangential plane. Let

$$
\mathfrak{U}(S)=\lim _{\mathfrak{G} \rightarrow 0} \inf _{\Omega_{\epsilon}} \sum \mu\left(S\left(\bar{G}_{i}-G_{i}\right)\right)
$$

where $\Omega_{\epsilon}$ is the class of sets of nonoverlapping open sets $G_{i}$ of diameter less than $\epsilon$ not meeting $A$ and such that $\Lambda^{m}\left(S^{0}-\sum G_{i}\right)=0$.

We shall show that:

THEOREM.

$$
\Lambda^{m} T=\mathfrak{A}(S) .
$$

As regards applying this to the Plateau problem for Hausdorff convex measure, ${ }_{c} \Lambda^{m}$, it suffices to note that for a surface minimising Hausdorff spherical measure, $\Lambda^{m}$, the two measures are equal and in any case

$$
\Lambda^{m}\left(S^{0}\right) \geqq{ }_{c} \Lambda^{m}\left(S^{0}\right) \geqq{ }_{c} \Lambda^{m}(T)=\Lambda^{m}(T)=\mathfrak{A}(S) \geqq \mu(L) .
$$

It is perhaps worth noting that $\mathfrak{A}(S)$ need not be as great as $\mu(A)$.

In order to prove that $\Lambda^{m}(T)=\mathfrak{A}(S)$ it suffices to cover nearly all of $S^{0}-T$ with small nonoverlapping open $G_{i}$ such that $\sum \mu\left(S\left(\bar{G}_{i}-G_{i}\right)\right)$ is small. We do so by applying the Vitale general covering principle [4] in two stages. At the first stage consider those points of $S^{0}$ which are restricted but not in $T$. By definition 


$$
\frac{\mu(s(P, r) S)}{r^{m}} \rightarrow 0 \quad \text { but } \quad \frac{\Lambda^{m} S S(P, r)}{r^{m}} \rightarrow 0
$$

so that there exist arbitrarily small spheres on which

$$
\frac{\mu\{s(P, r) S\}}{\Lambda^{m} S S(P, r)}<\epsilon .
$$

At the second stage we must consider the unrestricted points. To do so we need the full power of the main result of [3]; the "projection theorem."

According to this any $m$-set $E$ can be divided into two parts the restricted part $E_{R}$ and the unrestricted part $E_{u}$ and whereas the projection of $E_{R}$ onto almost any $m$ plane is a set of positive measure the projection of $E_{u}$ onto almost any $m$ plane is a set of measure zero.

In $n$-dimensional Euclidean space let $E(\theta, h)$ be the part of $E$ on the $(n-1)$-plane at distance $h$ from the origin and normal to the direction $\theta$. Write $R_{m} E=\Lambda^{m} E_{R}$. Then:

Lemma 1. For almost all $\theta$

$$
R_{m}(E) \geqq \int R_{m-1}(E(\theta, h)) d h .
$$

We can split $E$ up into its restricted and unrestricted parts. For a restricted set [2]

$$
R_{m}(E)=\Lambda^{m}(E) \geqq \int \Lambda^{m-1} E(\theta, h) d h \geqq \int R_{m-1} E(\theta, h) d h,
$$

and so we can confine ourself to the case of an unrestricted set $E$ when we must prove that

$$
\int R_{m-1}(E(\theta, h)) d h=0 .
$$

Let $\Pi_{m}$ be an $m$-plane through the origin, $\theta$ a vector in $\Pi_{m}$ and $h$ a real variable on $\theta$. Write $f\left(\theta, \Pi_{m}, h\right)$ for the $(m-1)$-dimensional Hausdorff spherical measure of the projection of $E(\theta, h)$ onto $\Pi_{m}$.

$$
\left.\int f\left(\theta, \Pi_{m}, h\right) d h=\Lambda^{m} \text { (Projection onto } \Pi_{m} \text { of } E\right)
$$

and therefore for almost all $\Pi_{m}$ the left-hand side is zero whenever $\theta \subset \Pi_{m}$.

Thus taking a fixed plane $\Pi_{n-m+1}^{0}$ 


$$
\int_{\theta \subset \Pi_{n-m+1}^{0}} d \theta \int_{\Pi_{m} \supset \theta} d \Pi_{m} \int f\left(\theta, \Pi_{m}, h\right) d h=0 .
$$

Hence for almost all $\theta \subset \Pi_{n-m+1}^{0}$

$$
\int_{\Pi_{m} \supset \theta} d \Pi_{m} \int f\left(\theta, \Pi_{m}, h\right) d h=0
$$

i.e.

$$
\int d h \int_{\Pi_{m} \supset \theta} d \Pi_{m} f\left(\theta, \Pi_{m}, h\right)=0
$$

so that for almost all $h: f\left(\theta, \Pi_{m}, h\right)=0$ for almost all $\Pi_{m} \supset \theta$, which implies $R_{m-1} E(\theta, h)=0$. This is true for almost all $\theta \subset \Pi_{n-m+1}^{0}$ and hence for almost all $\theta$.

Thus for almost all $\theta$ (1) holds and the lemma follows.

LemMa 2. If $\Gamma$ is an $(m-1)$ dimensional boundary of diameter $d$ and finite measure then

$$
\mu(\Gamma) \leqq \frac{d \mathfrak{X}(\Gamma)}{m}
$$

Choose a nonoverlapping set of open $G_{i}$ of diameter less than $\epsilon>0$ such that

$$
\sum_{1}^{\infty} \mu\left(\left(\bar{G}_{i}-G_{i}\right) \Gamma\right) \leqq \mathfrak{U}(\Gamma)+\epsilon
$$

and

$$
\Lambda^{m-1}\left(\Gamma-\sum_{1}^{\infty} G_{i}\right)=0 .
$$

Let $S_{i}$ be a surface of diameter less than $\epsilon$ with boundary $\supset H_{m-2}\left(\left(\bar{G}_{i}-G_{i}\right) \Gamma\right)$ and of measure $\mu\left(\left(\bar{G}_{i}-G_{i}\right) \Gamma\right)$. Then for any $N$ by [2]

$$
\mu(\Gamma) \leqq \mu\left(\Gamma-\sum_{1}^{N} G_{i}+\sum_{1}^{N} S_{i}\right)+\sum_{1}^{N} \mu\left(S_{i}+\bar{G}_{i} \Gamma\right)
$$

whence for large $N(3)$ and Lemma 7 of [2] give

$$
\mu(\Gamma) \leqq \frac{d(\mathfrak{A}(\Gamma)+2 \epsilon)}{m}+\frac{\epsilon \cdot 2 \Lambda^{m-1}(\Gamma)}{m}
$$

from which the lemma follows by letting $\epsilon \rightarrow 0$.

I now proceed by induction. Any closed set of finite linear measure can [5] be divided into a totally disconnected set plus a denumerable 
set of arcwise connected sets; so that our main theorem holds for $m=1$.

Suppose then that for $(m-1)$-dimensional surfaces $\Lambda^{m-1}(T)=\mathfrak{Y}(S)$ so that by Lemma 2

$$
\mu(\Gamma) \leqq \frac{d R_{m-1}(\Gamma)}{m}
$$

Take $G$ an open set containing $S_{u}^{0}$ the unrestricted part of $S^{0}$ and such that

$$
R_{m}\left(G S^{0}\right) \leqq R_{m}\left(S_{u}^{0}\right)+\epsilon=\epsilon .
$$

If $P \in S_{u}^{0}$ then by virtue of Lemma 1 we can find arbitrarily small cubes $\Delta(h) \subset G$ with side $2 h$ and centre $P$ such that if $\delta(h)$ denotes the surface of $\Delta(h)$ then

$$
\frac{2 R_{m}\left(S \Delta\left(h_{1}\right)\right)}{h_{1}} \geqq R_{m-1}\left(S \delta\left(h_{2}\right)\right)
$$

and $h_{1}>h_{2}>h_{1} / 2$.

By (4) above

$$
\mu\left(S \delta\left(h_{2}\right)\right) \leqq \frac{2 n}{m} R_{m}\left(S \Delta\left(h_{1}\right)\right) .
$$

By Vitale's theorem [4] we can now find a covering almost all of $S_{u}^{0}$ by a set $\phi$ of nonoverlapping $\Delta\left(h_{2}\right)$. The corresponding $\Delta\left(h_{1}\right)$ can be divided into a bounded set of classes of nonoverlapping cubes (cf. [4]) and each $\Delta\left(h_{1}\right) \subset G$; consequently by (5) and (6)

$$
\sum_{\phi} \mu\left(S \delta\left(h_{2}\right)\right)
$$

is small and the theorem follows.

\section{Bibliography}

1. E. R. Reifenberg, Parametric surfaces. II. Tangential properties, Proc. Cambridge Philos. Soc. 48 (1952), 46-69.

2. - Solution of the Plateau problem for m-dimensional surfaces of varying topological type, Acta Math. 104 (1960), 1-92.

3. H. Federer, The $(\phi, k)$ rectifiable subsets of $n$ space, Trans. Amer. Math. Soc. 62 (1947), 114-192.

4. A. S. Besicovitch, $A$ general form of the covering principle and the relative differentiation of additive functions. I, Proc. Cambridge Philos. Soc. 41 (1945), 103-110.

5. - On the fundamental geometrical properties of linearly measurable plane sets of points. II, Math. Ann. 115 (1938), 296-329.

University of Bristol, Bristol, ENGLAND 